\title{
Correction to: Predicting user visual attention in virtual reality with a deep learning model
}

\author{
Xiangdong $\mathrm{Li}^{1} \odot \cdot$ Yifei Shan ${ }^{1} \cdot$ Wenqian Chen ${ }^{1} \cdot$ Yue $\mathrm{Wu}^{1} \cdot$ Preben Hansen $^{2} \cdot$ Simon Perrault $^{3}$
}

Published online: 28 April 2021

๑) Springer-Verlag London Ltd., part of Springer Nature 2021

\section{Correction to: Virtual Reality}

https://doi.org/10.1007/s10055-021-00512-7

In the original publication, the spelling of fifth author was wrong. The correct spelling is 'Praben Hansen.' The original publication has been corrected.

Publisher's Note Springer Nature remains neutral with regard to jurisdictional claims in published maps and institutional affiliations.

The original article can be found online at https://doi.org/10.1007/ s10055-021-00512-7.

Xiangdong Li

axli@zju.edu.cn

Yifei Shan

shanyf@zju.edu.cn

Wenqian Chen

chenwenqian@zju.edu.cn

Yue Wu

yuewu0910@zju.edu.cn

Preben Hansen

preben@dsv.su.se

Simon Perrault

simon_perrault@sutd.edu.sg

1 College of Computer Science and Technology, Zhejiang University, Hangzhou 310027, China

2 Department of Computer Science and Systems, Stockholm University, Stockholm, Sweden

3 ISTD, Singapore University of Technology and Design, Singapore, Singapore 